\title{
A ultrassonografia das mãos no diagnóstico precoce e seguimento clínico de pacientes com Artrite Reumatóide
}

\author{
The ultrassonography of hands in early diagnosis and clinical follow-up of patients with \\ Rheumatoid Arthritis
}

\author{
Mariana Lima de Sousa ${ }^{1}$, Cláudia Tereza Lobato Borges²
}

\begin{abstract}
Resumo: A Artrite Reumatóide (AR) acomete aproximadamente 1\% da população mundial. Identificá-la como uma patologia de caráter aditivo, que afeta articulações periféricas em suas porções proximais e gera agravos incapacitantes com danos permanentes à qualidade de vida; bem como reconhecer que o tratamento precoce diminui sua progressão; impulsionaram pesquisadores na busca de um método adjuvante para diagnóstico antecipado e seguimento de atividade inflamatória: a ultrassonografia. Estudos sobre AR e voltados para análise das mãos foram depurados no intuito de contribuir para a discussão do tema e a implementação da ultrassonografia na rotina clínica. O desenvolvimento tecnológico recente proveu incrementos na qualidade da imagem e na resolução da ultrassonografia. Trata-se de uma poderosa ferramenta com uso novo na reumatologia e que apesar de operador dependente ganha prestígio por apresentar confiabilidade interobservadores ao captar áreas de sinovites de forma satisfatória e áreas de erosões ósseas com excelência e em estágios anteriores aos radiográficos, historicamente descrito na literatura médica como método de acompanhamento, que deve ser realizado no momento do diagnóstico e após um ano de doença, quando erosões, redução do espaço articular e subluxação podem ser detectadas. A avaliação do tecido musculoesquelético em escalas de cinza, em Doppler, com adição de contraste à imagem e padronização da técnica demonstra resultados equiparáveis à ressonância e superiores à radiografia. Por ser um exame mais barato, mais cômodo e que possibilita avaliar articulações em repouso e em movimento, a ultrassonografia em reumatologia tem sido considerada "uma extensão do exame físico".
\end{abstract}

Palavras-chave: Artrite Reumatóide; Ultrassonografia; Diagnóstico.

\begin{abstract}
Rheumatoid arthritis (RA) incidence is about $1 \%$ on the world's population. Identify it as an additive pathology that affects peripheral joints in its proximal portions and generates disabling diseases with permanent damage to the quality of life; as well as recognizing that early treatment limits its progression; stimulated researchers in the search for an adjuvant method for early diagnosis and follow-up of their inflammatory activity: the ultrasound. Studies on RA and aimed at hand analysis were debugged in order to contribute to the discussion of the subject and the implementation of ultrasonography in the clinical routine. Recent technological developments have resulted in improvements in image quality and resolution of ultrasonography. It is a powerful tool with new use in rheumatology and that, although dependent operator, gains prestige by presenting inter-observer agreement by capturing areas of synovitis satisfactorily and areas of bone erosion with excellence and in stages prior to radiography, historically described in the literature as a follow-up method, which should be performed at the diagnosis and after one year of disease, when erosions, reduction of joint space and subluxation can be detected. The evaluation of the musculoskeletal tissue in gray scales, in Doppler, with addition of contrast to the image and standardization of the technique demonstrates results comparable to the resonance and superior to the radiography. Because it is an economic and a more comfortable exam, that makes possible to evaluate joints at rest and in motion, ultrasonography in rheumatology has been considered "an extension of physical examination."
\end{abstract}

Keywords: Rheumatoid Arthritis; Ultrasonography; Diagnosis.

\footnotetext{
1 Universidade CEUMA, Medicina (Maranhão, Brasil)

2 Universidade CEUMA, Prof. Reumatologista (Maranhão, Brasil)
}

Autor correspondente: Mariana Lima de Sousa Universidade CEUMA, Campus Renascença. Endereço: Rua Josué Montello, no1, Renascença II - CEP 65.075-120 - São Luís/MA. Telefone: (98)3214-4277. E-mail: mari_lima4@hotmail.com 


\section{Introdução}

A Artrite Reumatóide acomete cerca de $1 \%$ da população em todo o mundo $^{1,4}$. É uma doença sistêmica inflamatório-degenerativa crônica e progressiva mediada por auto anticorpos que afetam principalmente a membrana sinovial e que pode levar à destruição óssea e cartilaginosa ${ }^{2}$. Logo, envolve sinais e sintomas constitucionais como: febre, adinamia, mal-estar e emagrecimento; e reações localizadas como: inflamação e proliferação da sinóvia, destruição do tecido cartilaginoso articular, erosão óssea e consequente estreitamento da porção justa-articular ${ }^{3}$.

Dentre os fatores relacionados com sua etiologia a herança genética é positiva em $60 \%$ dos casos, com aumento de incidência entre parentes de primeiro grau. O primeiro loci genético descrito pertence ao antígeno leucocitário humano, o HLA-DRB1, hoje outros 30 adicionais já são conhecidos. Outros fatores considerados desencadeadores são o componente hormonal, o tabagismo e algumas infeções virais por Epstein-Barr Vírus e Parvovírus B-19 e infecções bacterianas por $M$. tuberculosis, E. coli e P. mirabilis ${ }^{4}$.

A fisiopatologia da Artrite Reumatóide é fruto da combinação da atividade do sistema imune inato e do sistema imune adaptativo, que desencadeia uma desordem entre a atuação de citocinas pró-inflamatórias (TNF-alfa, IL-1 e IL-6) e citocinas antiinflamatórias (IL-4 e IL-10). A membrana sinovial torna-se espessada, há proliferação vascular e o tecido inflamado adquire potencial de invadir e degradar 0 tecido conjuntivo por liberação de enzimas denominadas metaloproteinases, esse tecido é conhecido como pannus. Porém, muitos dos mecanismos envolvidos ainda seguem não inteiramente esclarecidos ${ }^{4}$.

Dados epidemiológicos revelam que esta patologia afeta três vezes mais mulheres que homens quando se considera sua epidemiologia à nível global, principalmente entre a quarta e a sexta décadas de vida. Entretanto estudos no Brasil apontaram uma difusão da doença com proporção de um homem para cada seis mulheres afetadas. Sua prevalência aumenta com a idade e incide sobre todas as raças com graus variados de agressividade e frequência, diminuindo a expectativa de vida dos pacientes em cinco a dez anos ${ }^{2}$.

$\mathrm{Na}$ forma clássica, a Artrite Reumatóide afeta pequenas articulações periféricas com aspecto simétrico e aditivo, com capacidade de evolui para incapacidade física e dano significativo na qualidade de vida ${ }^{5}$. Um exemplo é que na progressão da doença para os punhos, o tecido sinovial aumentado pode comprimir o nervo mediano e causar Síndrome do Túnel do Carpo ou até mesmo invadir tendões extensores e provocar sua ruptura e perda funcional da mão ${ }^{3}$.

Para pacientes com mais de um ano de atividade da doença, o diagnóstico é descrito nos Critérios de Classificação da Artrite Reumatoide de 1987. Devem estar presentes por pelo menos seis semanas: rigidez matinal com duração de pelo menos 1 hora; artrite de três ou mais áreas, sendo pelo menos três constituídas de edema de partes moles ou derrame articular; artrite de articulações das mãos (interfalangeanas proximais, 
metacarpofalangeanas e punho); e artrite simétrica. $O$ nódulo reumatóide pode estar presente $\mathrm{O}$ Fator Reumatóide e a radiografia das mãos fecham os 7 critérios, dos quais 4 já indicam $\mathrm{AR}^{4}$.

O diagnóstico com menos de um ano de atividade de doença é através dos Critérios da American College of Rheumatology e da European League Against Rheumatism é de 2010 , se baseia no acometimento articular quantitativo e qualitativo; na presença de Fator Reumatóide (FR) e anticorpo AntiPepitídeo Citrulinado Cíclico (anti-CCP) positivos; em valores alterados de proteína C-reativa (PCR) e Velocidade de Hemossedimentação (VHS); e no tempo de duração dos sintomas, sendo necessário pontuar igual ou superior a seis pontos nos critérios para definição diagnóstica ${ }^{4}$.

É válido citar que anticorpo antiCCP está positivo em 60 a $70 \%$ dos pacientes no momento do diagnostico de Artrite Reumatóide. Já o FR está presente em apenas $50 \%$ dos casos e em 20 a $35 \%$, o FR só positivará nos primeiros seis meses após sintomas clínicos, ou seja, quando já estabelecido o diagnóstico ${ }^{3}$.

As medidas terapêuticas na Artrite Reumatóide estão direcionadas para atingir a mínima atividade da doença ou remissão ${ }^{3}$. Martins et. Al. (2016) defendem ainda que detectar parâmetros, novos técnicas e seus achados, com capacidade de indicar processos de atividade e remissão e prognóstico da doença é de substancial importância para conduzir o tratamento adequado.

O diagnóstico diferencial deve ser realizado com osteoartrite nodal, doença gonocócica disseminada, hepatite viral crônica, Lupus Eritematoso Sistêmico, Síndrome de Sjögren, miosites inflamatórias, artrite psoriásica, devendo sempre direcionar para o tipo de paciente: mulher jovem, indivíduo sexualmente ativo ou idosos ${ }^{4}$.

$\mathrm{Na}$ fase ativa da doença o tratamento é com anti-inflamatórios não hormonais em doses plenas e esse tratamento pode se estender por semanas. Devido à duração do tratamento, deve estar associado um inibidor da bomba de próton. O corticoide em baixas doses é usado enquanto não agem as drogas modificadoras do curso da doença. Caso haja indicação de uso por tempo prolongado indicar uso de Cálcio, Vitamina D e Bifosfonados ${ }^{4}$.

Agentes modificadores do curso da doença (AMCD) interferem na progressão clínica. O esquema deve compor na primeira etapa: um único AMCD sintético (Metotrexate, Sulfassalazina, Leflunomida e Hidroxicloroquina) por 3 meses, se necessário, nos 3 meses consecutivos, acrescentar outro AMCD sintético. Se não houver controle da doença, seguir para segunda etapa: associar AMCD biológico (Tofacitinibe, Agentes com ação antifator de necrose tumoral, Rituximabe, Abatacepte e Tocilizumabe) ao sintético. A terceira etapa descreve a troca entre biológicos com melhor resposta no organismo do doente ${ }^{4}$.

O controle da atividade da doença é historicamente realizado com radiografias das mãos que por vezes estão normais ao diagnóstico e devem ser repetidas após um ano ou mais, período em que as erosões, redução do espaço articular e subluxação podem ser detectadas por 
esse exame. Nesse ponto já há destruição óssea e deformidade articular irreparável.

A última década assistiu o emprego da ultrassonografia (US) nas doenças reumatológicas com grandes resultados na avaliação articular do paciente com AR. Pesquisadores sugerem se tratar de uma ferramenta sensível para detecção e seguimento clínico da atividade inflamatória no paciente, uma grande oportunidade de prevenir o dano articular irreversível?.

Esse novo elemento abordado em artigos científicos aqui será depurado no intuito de contribuir para sua discussão enquanto aplicação na $A R$ e sua implementação na rotina clínica. Portanto, o objetivo dessa revisão é estudar a AR e aspectos novos no diagnóstico precoce e controle de atividade da doença.

Com especial enfoque na compreensão da importância epidemiológica para a suspeição do diagnóstico, em contribuir para produção científica sobre o tema, estabelecer 0 benefício do diagnóstico precoce e controle de atividade da doença e indicar a implementação da ultrassonografia na rotina clínica.

\section{Material e método}

Este trabalho está fundamentado em um compilado de pesquisas bibliográficas. É fruto da análise dos conceitos de livros e artigos científicos. Trata-se de uma investigação focada em tema bem definido e após identificação, seleção e avaliação das evidências relevantes disponíveis, traz um amplo conjunto de informações sobre o tema ${ }^{8}$.
Para esse estudo, foram interpretados 23 materiais dentre artigos científicos e livros. É válido destacar que 10 estudos investigaram a qualidade dos diversos métodos ultrassonográficos na interpretação de achados musculoesqueléticos, outros 5 estudos avaliaram a concordância interobservadores para o método e um estudo avaliou a progressão da doença ultrassonografiacamente e concomitante a realização de tratamento longitudinalmente por um período de 7 anos.

Ao iniciar a pesquisa, empreendeuse também uma vasta revisão bibliográfica sobre Artrite Reumatóide, no que tange epidemiologia, fisiopatologia, quadro clínico, diagnóstico e tratamento. Diversas fontes contribuíram para constituição deste estudo.

\section{Revisão de literatura}

Até o momento, o exame de imagem consagrado nas literaturas ainda é a radiografia das mãos ${ }^{3}$ que por muitos anos foi citado nas bibliografias como o grande exame a ser solicitado para os pacientes, em contraponto à Ressonância Nuclear Magnética que é um método caro e, principalmente àquela época, não tão fácil de se lançar mão devido à seu acesso mais difícil.

Porém, as máquinas de Ultrassonografia de alta qualidade têm demonstrado evidente vantagem devido à boa resolução da imagem e a percepção espacial da área articular, o que proporciona ao reumatologista identificar a inflamação e os danos estruturais, 
monitorar as doenças e prever as respostas terapêuticas ${ }^{9}$.

A sinovite é a lesão mais precoce capaz de ser graduada pela Ultrassonografia, uma alteração que pode se apresentar com leve proliferação ou exsudação de uma sinóvia que quando saudável é imperceptível ${ }^{5}$.

Para Arend (2013), é classificada pela gravidade através da ultrassonografia em leve, moderada e exuberante e nesses pacientes são avaliados a distensão da capsula articular pelo tecido hipoecogênico e a pobre compressão tecidual do conteúdo proliferado com o transdutor.

Outra forma de realizar o estudo articular na sinovite é pelo Power Doppler (PD) colorido. Essa técnica determina um realce da vascularização na sinóvia que passa a se manifestar com hiperfluxo na fase ativa da doença. Além disso, é possível a percepção dos padrão de resistência: quando baixa, sinaliza uma fase aguda; e quando alta, sinaliza uma fase crônica ativa ${ }^{5}$.

Um ponto negativo é a ausência quantitativa ou qualitativa de um ponto de corte estabelecido que caracterize alta ou baixa resistência através de um fluxo captado pelo transdutor, encontrar este valor é o objetivo de muitos estudos científicos. A única certeza entre os autores é que o fluxo diastólico nulo ou reverso é um indicador de alta resistência ${ }^{5}$.

Bisi et al. (2014) desenvolveram um estudo no qual analisaram 1.380 articulações de pacientes com Artrite Reumatóide com o objetivo de mensurar a concordância interobservadores. Para delinear o estudo dois reumatologistas foram treinados por um ano e os resultados obtidos foram então correlacionados através do kappa ponderado. A confiabilidade interobservadores foi de razoável a boa para sinovites em escalas de cinza, no Power Doppler de moderada a excelente e para a presença de erosões ósseas, de boa a excelente.

Outro benefício da Ultrassonografia é a utilização do histograma de imagens coloridas para melhor visualização de danos ecotexturais, essa técnica apresentou melhor visualização das estruturas através de uma tecnologia moderna que já permite retratar detalhes de milímetros ${ }^{11}$.

Percebe-se que a avaliação da atividade sinovial expressa pelo aumento da vascularização, pela destruição da cápsula articular, pelas erosões e pela proliferação tecidual é extremamente importante para o diagnóstico e avaliação do efeito do tratamento ${ }^{2,13}$. E o exame de imagem com boa acurácia para avaliar esses achados por anos foi somente a Ressonância Nuclear Magnética ${ }^{14}$.

Quando a Ultrassonografia foi comparada à Ressonância Nuclear Magnética no âmbito do diagnóstico descobriu-se um resultado benéfico em relação à detecção da artrite inflamatória na fase inicial, em especial naqueles com doença soronegativa. No âmbito do prognóstico, tanto a escala de cinza quanto o Power Doppler apontaram a sinovite e previram a progressão da erosão, resultado compatível com o obtido na Ressonância Nuclear Magnética ${ }^{16}$.

Para Mendonça (2014), a Ultrassonografia demonstra os processos inflamatórios por meio de escala de 
matizes e de contrastes e estabelece as características e a extensão de estruturas acometidas. Reflete a fase evolutiva e o grau de agressividade, para o autor acima, descritos em 4 fases: ausente, leve, moderado e importante, ao considerar a ecogenicidade e distensão da capsula articular.

Os pesquisadores do Colégio Americano de Reumatologia avaliaram 150 articulações entre as metacarpofalângicas e as interfalângicas proximais em mais um estudo para também delimitar a concordância entre dois observadores. Nele, os resultados obtidos foram em $91 \%$ dos exames como concordantes em haver erosões ósseas, em $86 \%$, consoantes para sinovite, em $79 \%$, para derrames articulares e em $87 \%$, para sinovites no PD. Os autores assentem que a ultrassonografia pode melhorar a avaliação de pacientes com Artrite Reumatóide por radiologistas e reumatologistas ${ }^{17}$.

Por fim, a RM é um método caro de obtenção de imagem dependente da disponibilidade do aparelho, ainda que a imagem seja com excelente resolução ${ }^{6}$. Do outro lado tem-se a Ultrassonografia, uma técnica não invasiva, rápida, de baixo custo, com maior tolerabilidade, que possibilita escanear múltiplas articulações em repouso e em movimento e correlacionar diretamente os achados clínicos e de imagem ${ }^{15,10}$. Por isso é considerada pelo Mendonça (2014) "uma extensão do exame físico".

Assim denominada por sua capacidade de incrementar a atuação clínica, auxiliando de forma prática, rápida e indolor, associando a isso os aspectos de anamnese e de exame físico para detecção de achados próprios da doença e monitorar a progressão das lesões.

\section{Discussão}

Como exposto acima, a ultrassonografia revela uma nova perspectiva ao se mostrar como método adjuvante para o diagnóstico de Artrite Reumatóide e o acompanhamento do ciclo de atividade e de remissão da doença.

$\mathrm{Na}$ busca de artigos que avaliavam a concordância interobservadores, embora todos concordem que 0 treinamento de médicos e sua capacitação determina uma maior uniformidade nos laudos das imagens, apenas 5 estudos relatando essa experiência contribuíram para a confecção desse trabalho.

Dentre os pontos positivo deste trabalho, pode-se citar a as minúcias da avaliação do tecido musculoesquelético em escalas de cinza, pelo método Power Doppler, com adição de contraste à imagem e padronização da técnica que demonstram resultados equiparáveis à ressonância e superiores à radiografia ${ }^{21,22}$.

Também, as imagens em escalas de cinza com alta resolução, quando combinadas com a análise pelo Power Doppler, que tornam possível verificar o fluxo sanguíneo de baixo volume, abrindo novos caminhos para detectar e classificar a inflamação articular leve ${ }^{9}$. 


\section{Conclusões}

Realizar o diagnóstico precoce e, em consequência, o acompanhamento adequado do tratamento de pacientes com Artrite Reumatóide pode evitar ou diminuir a dor, o edema articular, o risco de inflamação persistente, a progressão do pannus, a evolução do dano ósseocartilaginoso e incapacidades irreversíveis ${ }^{18}$.

A US do tecido musculoesquelético se mostrou capaz de examinar áreas de atividade inflamatória das sinóvias, principalmente expressa pelo aumento da vascularização, de perceber a destruição da capsula articular e as erosões ósseas ${ }^{19,20,13}$

Além disso, apresenta vantagens em relação a outras ferramentas de imagem, a Radiografia, apesar de um método barato, de fácil acesso, pouco incômodo, de rápida realização, não mostra alterações precoces da doença. ${ }^{9}$.

No outro extremo, tem-se um exame de imagem bastante requintado, caro e de difícil acesso para grande parte da população brasileira, a Ressonância Nuclear Magnética que permite visualizar pequenas alterações teciduais com a característica de ser um exame mais incômodo e que estuda articulações apenas em repouso ${ }^{9}$.

Para Perricone ${ }^{23}$ e Fillipucci ${ }^{7}$ um momento bastante esperado pelos reumatologistas foi poder lançar mão dessa nova ferramenta, a Ultrassonografia, para o benefício do paciente. Por todos os resultados acima, já está indicada a implementação da ultrassonografia na rotina clínica ${ }^{4}$.
As recomendações (critérios) para o diagnóstico da Artrite Reumatóide ainda não foram modificadas, seguem as mesmas pelo último Consenso da Sociedade Brasileira de Reumatologia de 2011, porém, como frisado anteriormente, a ultrassonografia já uma realidade, seu uso não refletirá imprudência, e deve-se lançar mão dessa ferramenta quando possível visando o benefício do paciente.

\section{Conflito de interesses}

A autora e a orientadora afirmam não haver conflito de interesses.

\section{Referências}

1. Alamanos $Y$, Voulgari $P V$, Drosos $A A$. Incidence and prevalence of rheumatoid arthritis, based on the 1987 American College of Rheumatology criteria: a systematic review. Semin Arthritis Rheum, v. 36, p. 182-8, 2006.

2. Mota, LMH, et al. 2011 Consensus of the Brazilian Society of Rheumatology for diagnosis and early assessment of rheumatoid arthritis. Rev. Bras. Reumatol., São Paulo, v. 51, n. 3, p. 207-219, June 2011

3. Imboden, J.B.; Hellmann, D.B..; Stone, J.H. Current reumatologia: diagnóstico e tratamento. 3. ed. São Paulo, SP: McGrawHill Education, 2014.

4. Martins MA, et al. Clínica médica : Doenças Endócrinas e Metabólicas, Doenças Ósseas, Doenças Reumatológicas. Vol.5 Barueri: Manole, 2016.

5. Goeldner I, et al. Artrite reumatoide: uma visão atual. J.Bras. Patol Med. Laboratório Rio de Janeiro, v. 47, n.5, p.495-503, out. 2011.

6. Arend CF. Ultrasonography in rheumatoid 
arthritis: what rheumatologists should know. Rev. Bras. Reumatol., São Paulo, v. 53, n. 1, p. 94-100, Feb. 2013.

7. Fillipucci et al. Ultrasound imaging for the rheumatologist. Clin Exp Rheumatol. 2006 vol. 24 (pág. 1-5).

8. Köche JC. Fundamentos de metodologia científica: teoria da ciência e iniciação à pesquisa. Petrópolis, RJ : Vozes, 2011.

9. Kang $T$, et al. Value of Ultrasound in Rheumatologic Diseases. J Korean Med Sci. v.4, p.497-507. apr 2013

10. Bisi $M C$, et al. Ultrassonografia articular: confiabilidade interobservadores em artrite reumatoide. Rev. Bras. Reumatol., São Paulo , v. 54, n. 3, p. 250-254, June 2014.

11. Mendonca JA. Ultrasound color histogram assessment allows better view of echotexture damage. Rev. Bras. Reumatol., São Paulo , v. 57, n. 1, p. 88-91, Feb. 2017

12. Mota LMH, et al.2011 Consensus of the Brazilian Society of Rheumatology for diagnosis and early assessment of rheumatoid arthritis. Rev Bras Reumatol. 2011 May-Jun;51(3):199-219.

13. lagnocco, et al. Monitoramento clínico e ultrassonográfico da resposta ao tratamento com Adalimumabe na Artrite Reumatóide. The Journal of Rheumatology. v.35, i.1, p. 35-40; Jan 2008.

14. Junior, WCT, Rolim R, Kakehasi AM. Imagens de ressonância magnética na artrite reumatoide. Rev Bras Reumatol. v. 51 p. 629641. 2011.

15. Mendonça JM. Ultrassonografia em Reumatologia: uma extensão do exame físico. Thieme Revinter, Campinas, SP. 2014.

16. Tan YK, Østergaard M, Conaghan PG. Imaging tools in rheumatoid arthritis: ultrasound vs magnetic resonance imaging, Reumatologia, v. 51, Edição suppl_7, p. vii36vii42. dez 2012.

17. Szkudlarek M, et al. Interobserver agreement in ultrasonography of the finger and toe joints in rheumatoid arthritis. Arthritis \& Rheumatology. V.48, p. 955-962. April 2003.

18. De Santana, et al; Avaliação da capacidade funcional em pacientes com artrite reumatoide: implicações para a recomendação de exercícios físicos. Rev Bras Reumatol. 54(5):378-385, 2014.
19. Fernandes EA, et al. Ultrasonography in rheumatoid arthritis: applicability and expectation. Rev. Bras. Reumatol., São Paulo. v. 48, n. 1, p. 25-30, Feb. 2008.

20. Ellegaard K, et al. Ultrasound colour Doppler measurements in a single joint as measure of disease activity in patients with rheumatoid arthritis-assessment of concurrent validity. Rheumatology, v. 48, i. 3, 1 p.254-257 March 2009.

21. Scheel, et al. Estudo prospectivo de acompanhamento de 7 anos comparando radiografia, ultrassonografia e ressonância magnética em articulações de artrite reumatoide. Ann Rheum Dis , v. 65 p. 595600. 2006

22. Gutierrez, et al. Confiabilidade interobservador da ultra-sonografia de alta resolução na avaliação de erosões ósseas em pacientes com artrite reumatoide: experiência de um programa intensivo de treinamento dedicado. Reumatologia, vol. 50, ed. 2, 1, p. 373-380. Fev 2011.

23. Perricone $\mathrm{C}$, et al. Avaliação ultrassonográfica de 6 articulações: um método válido, sensível a mudanças e viável para avaliar inflamação articular em AR, Reumatologia, vol.51, n.5, p. 866 a 873. maio 2012. 